\title{
有机化学实验教学内容改革笔谈
}

\author{
强根荣 ${ }^{*}$, 王海滨 \\ 化学化工国家级实验教学示范中心(浙江工业大学), 杭州 310014
}

\begin{abstract}
摘要: 有机化学实验是化学、化工、制药及其他近化学类专业学生必修的一门基础实验课程, 对增强学生动手能力、培 育创新思维有不可替代的作用。本文以八个具体的实验为实例, 介绍了随着时代变迁和人才培养规格的变化, 有机化学 实验课程建设、教学内容改革的情况, 从早期简单的过程模仿、性质鉴定、基本操作实验, 发展为现有的 “基础规范性 实验-综合设计性实验-研究探索性实验”三层次实验, 并经实践检验行之有效, 使实验教学形式更丰富、内容更充实、 手段更新颖, 更加有利于学生的多样化成才, 教学质量和教师的教研水平得到极大提高。
\end{abstract}

关键词: 有机化学实验; 实验教学; 教学改革

中图分类号: G642.1; O6

\section{Reforming the Experimental Teaching Content for Organic Chemistry}

\author{
Genrong Qiang *, Haibin Wang \\ National Demonstration Center for Experimental Chemistry and Chemical Engineering Education (Zhejiang University of \\ Technology), Hangzhou 310014, China.
}

\begin{abstract}
Organic chemistry experiment is a basic compulsory experimental course for students majoring in chemistry, chemical engineering, pharmacy, and other modernized majors. It enhances students' practical abilities and cultivates innovative thinking. This article uses eight specific experiments as examples for the construction of organic chemistry experiment courses and the reform of teaching content to satisfy changes in talent training specifications. Experimental teaching has developed from the early simple simulation process, identification of nature, and basic operational experiments to the existing three-level "basic normative experiment-comprehensive design experimentresearch exploratory experiment". This involves a more in-depth experimental teaching method, of which the content is more substantial and the methods novel; this is conducive to broadening the knowledge of students. This method has greatly improved the quality of teaching and the level of teaching and research by teachers.
\end{abstract}

Key Words: Organic chemistry experiment; Experimental teaching; Teaching reform

有机化学实验是我校化学、化工、制药及其他近化学类专业学生必修的一门基础实验课程。现 有教学对象每届 20 个专业、36个班级、约 1100 名学生, 教学总时数为 64 , 学分为 2 , 大二年级分两学 期开设。从1953年建校开课, 设立有机化学实验课程以来, 随着时代变迁和人才培养规格的变化, 课程建设、教学内容发生了翻天覆地的变化, 从早期简单的过程模仿、性质鉴定、基本操作实验, 发展为现有的 “基础规范性实验-综合设计性实验-研究探索性实验” 三层次实验。多年来, 实验中

收稿: 2021-09-24; 录用: 2021-09-27; 网络发表: 2021-11-03

“通讯作者, Email: qgr@zjut.edu.cn

基金资助：浙江省本科高校 2020 年度省级一流课程认定(浙教办函〔2021) 195 号) 
心教师从 “以本为本” 的教学理念出发, 根据新时代人才培养的要求, 在教学内容方面大刀阔斧地 进行改革, 并经实践检验行之有效, 已将“有机化学实验”课程建设成为省级一流课程。

\section{1 有机化学实验教学内容改革的基本遵循}

我校有机化学实验的教学对象都是工科类专业, 毕业去向大都是继续深造、研发机构或生产企 业, 因此, 教学内容的设置要有利于学生在掌握实验基本理论、基本知识的基础上, 更多地增强学 生的实际动手能力、培育创新思维, 秉承并发扬光大浙工大学生 “下得去、上得来、用得上” 的优 良品质, 这也是我们实验教学改革和实验内容建设的基本遵循。特别是在 “新工科” 大背景下, 我 们将有机化学课程实验教学纳入理工融合、基础与专业有机衔接的 “三阶段(学科基础实验-专业基 础实验-专业实验和工程实训)、四层次(基础规范性实验-综合设计性实验-研究探索性实验-创新实 践)、一体化” 的化学化工实验教学体系 ${ }^{[1-4]}$, 着力培养学生把知识运用于实践的能力, 促使 “科学原 理探索-工程技术基础训练-单元操作组合运用-工程实践”的融会贯通 ${ }^{[5,6]}$ 。

\section{2 有机化学实验教学内容改革实践}

由于受专业性质和实验教学时数的限制, 我们的教学内容改革只能在受限的时数内 “推陈出新”, 因此, 一方面不再单独开设基本操作实验和性质实验, 将这些实验都融入到合成实验中; 另一方面, 研究开发反应时间较短而基本操作丰富的实验, 再揉入新的操作和新的内容。以下实例展示了我校 有机化学实验教学内容改革的来龙去脉, 也为其他院校教学改革提供参考。

实例一：乙酰苯胺的制备及重结晶 ${ }^{[7-9]}$

在早期的实验教学中, 为了学习重结晶基本操作, 我们从企业购买了含有菜的废料, 用 $70 \%$ 的 乙醇作溶剂, 通过重结晶操作提纯蒜, 让学生学会用有机物作溶剂进行重结晶操作的方法。随着时 代的发展和企业撤并关停, 一方面实验原料不再容易购买, 另一方面学生对重结晶操作的实际应用 不太清楚, 为此, 我们在乙酰苯胺的制备实验中增加重结晶操作, 用水作溶剂对乙酰苯胺的粗产品 进行提纯, 经干燥后进行红外光谱、质谱鉴定, 不再单独开设 “粗菜的提纯” 实验。

实例二: 二苯基乙二酮的制备及薄层色谱

薄层色谱方法在有机合成实验中有非常重要的用途, 如: 鉴定化合物、监测跟踪反应进行的程 度、分离与精制化合物、检测其他方法的分离纯化效果、作为柱色谱的先导粗略判断混合物中各组 分的相对浓度等等。早期的实验教学中, 我们单独开设 “薄层色谱” 基本操作实验, 用苏丹红溶液、 靛酚蓝溶液以及它们的混合物作为三个样品, 学生制作薄层板后, 在板上展开, 计算比移值即可。 这种做法在实际应用过程中的局限性非常明显, 学生不太清楚薄层色谱的真正用途。感受最深的是 我们学校的学生在2008年第一次参加全国大学生化学实验邀请赛, 在赛场的实验台上放有硫酸的展 开缸, 实际上已经很明显地提示学生用薄层色谱来跟踪反应进程, 但我们的学生不是很清楚如何使 用。痛定思痛后, 我们认识到学生做实验时仅知道实验原理是不够的, 必须亲自动手才能起到真正 的效果, 于是在 2009 年即着手改革薄层色谱操作实验, 改变了原有的做法, 将薄层色谱操作应用到 二苯基乙二酮的合成过程中。该实验反应时间较短, 既保证了在原有教学时数内完成实验, 又 “真 刀真枪” 地将薄层色谱操作应用到实际过程中, 让学生感受实际的应用过程。二苯基乙二酮粗品采 用 $80 \%$ 乙醇重结晶提纯 ${ }^{[10,11]}$, 增加了有机物作溶剂进行重结晶操作的训练, 区别了乙酰苯胺用水作 溶剂重结晶的操作。

实例三: 乙酰二茂铁的制备及柱色谱分离

与理科类专业实验教学相比较, 工科类专业有机化学实验教学的学时数明显偏少, 因此, 我们 原先并没有开设柱色谱相关实验。为训练学生安装、使用电动摚拌基本操作的能力, 我们开设有 “邻 叔丁基对苯二酚的制备” 实验, 在实际的运行过程中, 发现其主要缺陷是实验用二甲苯作溶剂, 实 验室气味很大, 环境不太友好。基于上述两种因素, 同时服务于学生参加各类竞赛及深造、工作的 
实际需求, 我们开发了 “乙酰二茂铁的制备及柱色谱分离” 实验, 将电动摚拌操作应用于乙酰二茂 铁的合成中, 并增加了柱色谱分离粗品的实验, 在不增加学时数的情况下, 既保证原有操作不丢失, 又增加了新的操作 ${ }^{[12,13]}$ 。

实例四: 苯甲酸(微波)与苯甲酸乙酯的制备及减压蒸馏

早期的实验中采用购买来的试剂苯甲酸乙酯作原料进行 “减压蒸馏” 操作实验, 成本高、效果 差。研发、开设的新实验包含的基本操作很多, 有微波操作、分水器的使用、加热回流、萃取洗涤、 液体物质干燥、低沸点易燃有机溶剂的蒸馏、减压蒸馏等等, 实验综合性较强。另外, 作为一个连 续性实验, 学生先要合成苯甲酸, 再通过酯化反应合成苯甲酸乙酯, 最后产品要通过减压蒸馏来精 制, 学生必须全面地考查各个实验环节, 注重实验的各个细节, 才能得到满意的实验结果。本实验 着重改革了以下几个方面:

(1) 苯甲酸的合成。苯甲酸的合成方法有很多, 我们原本是用甲苯作原料、用高锰酸钾作氧化剂 制备而成的, 反应时间很长, 大约需要 $4-5 \mathrm{~h}$ 完成实验。实验过程中, 将电动搅拌安装好后基本没有 其他工作可以做了, 学生觉得枯燥乏味。从2004年第四届全国大学生化学实验激请赛的竞赛内容得 到启发, 我们用苯甲醇作原料, 采用微波加热来合成苯甲酸, 反应时间缩短到 $20 \mathrm{~min}$ 。其中苯甲醇 来源于另一个实验 “苯甲醇与苯甲酸制备” 的产品, 学生实验产品得到循环利用, 并包括微波加热 这一新型的实验手段, 起到节能高效的作用, 充分地体现了化学实验蕴含的绿色化学理念, 保障了 学生实验的绿色化, 将 “思政” 元素融入到实验教学中。

(2) 苯甲酸乙酯的合成。苯甲酸乙酯的合成采用共沸脱水的方法, 通过分水器将反应过程中产 生的水带出反应体系。我们原本采用苯作带水剂, 毒性较大, 学生做实验时缩手缩脚, 近几年, 经 过摸索改用环己烷作带水剂, 极大地提高了实验的绿色化程度 ${ }^{[14]}$ 。另外在实验方案中, 原本反应开 始时就安装了分水器, 由于学生初次使用该仪器, 操作不熟练, 分水器放水不当, 往往是放出的水 太多, 导致大量环己烷滞留在分水器中, 影响了反应的进行, 苯甲酸反应不完全, 在后期的减压蒸 馏时, 蒸馏瓶及克氏蒸馏头上留有很多的固体苯甲酸, 毛细管堵塞。我们在改革中先直接加热回流 $30 \mathrm{~min}$, 等反应基本平衡后再装上分水器回流脱水, 反应就比较完全, 实验效果大大提高。

(3) 减压蒸馏。苯甲酸乙酯通过减压蒸馏提纯, 原本采用玻璃水冲洜(后改用自制的铜质水冲洜, 俗称 “水老鼠”)作减压设备用, 其最大的优点是节省空间(我们现在做抽滤时还是用这个 “水老鼠”), 缺点是学生做完实验后对整个减压蒸馏体系不了解, 为此, 我们自行设计制造了减压蒸馏小推车, 包括完整的缓冲瓶、冷阱、气体干燥塔(三个)、低真空测压仪、真空泵等。

实例五：2-甲基-2丁醇的制备

本实验用格氏反应(Grignard Reaction)制备2-甲基-2丁醇, 实验中采用磁力摚拌, 以区别于其他 实验的电动摚拌。实验过程中粗产物经洗涤后, 要用乙醚萃取。根据分配定律, 用相同量的溶剂, 分几次萃取要比一次萃取效率高。因此, 一般情况下, 分2-4次萃取, 就可以把绝大部分被萃取物萃 取出来。但学生做实验时, 不太理解这个原理。我们给出以下题目, 让学生去计算, 促使学生更加 深刻地理解其中的道理。

例题: 溶有少量物质 $\mathrm{A}$ 的水溶液 $30 \mathrm{~mL}$, 现用乙醚萃取回收该物质 $\mathrm{A}$ 。已知物质 $\mathrm{A}$ 在水和乙醚中 的分配系数为 $1: 5$, 试问用 $30 \mathrm{~mL}$ 乙醚一次萃取, 或用 $30 \mathrm{~mL}$ 乙醚分两次萃取, 它们的萃取效率如何?

解答: 用 $30 \mathrm{~mL}$ 乙醚一次萃取。留在水中的量为:

$$
m_{1}=m_{0}(1 / 5 \times 30) /(1 / 5 \times 30+30)=1 / 6 m_{0}
$$

效率为:

$$
(1-1 / 6) m_{0}=5 / 6 m_{0}=83.3 \%
$$

改用 $30 \mathrm{~mL}$ 乙醚分两次萃取。第一次萃取后留在水中的量为:

$$
m_{1}=m_{0}(1 / 5 \times 30) /(1 / 5 \times 30+15)=2 / 7 m_{0}
$$

再经第二次萃取后留在水中的量为: 


$$
m_{2}=m_{1}(1 / 5 \times 30) /(1 / 5 \times 30+15)=4 / 49 m_{0}
$$

因此, 萃取效率为:

$$
(1-4 / 49) m_{0}=45 / 49 m_{0}=91.8 \%
$$

实例六: 环已烯的制备

学生在做了蒸馏与分馏的基本操作实验后, 我们采用半微量仪器开设 “环己烯制备” 实验, 有 利于学生进一步掌握蒸馏、分馏的原理, 更加仔细地观察实验现象。本实验采用边反应边蒸出环己 烯(与水的共沸物)的办法, 几个温度节点一定要让学生要把握好。

第一个节点是环己醇与水的共沸点: 刚开始反应时, 反应还没有开始, 反应瓶中有环己醇和水, 温度太高, 柱顶温度必然上升到 $97.8^{\circ} \mathrm{C}$ 左右。

第二个节点是环己烯与水的共沸点: 随着反应的进行, 环己烯的生成量增加, 反应瓶中有环己 醇、环己烯和水, 柱顶温度必然稳定在 $71^{\circ} \mathrm{C}$ 左右。

第三个节点是环己烯的沸点: 反应一段时间后, 环己醇基本作用完全, 反应瓶中的水也基本带 出, 因此, 此时如果反应瓶中还有环已烯, 柱顶温度一般稳定在 $83^{\circ} \mathrm{C}$ 左右, 超过这个温度表示环己 烯基本蒸完了。

实例七: 肉桂酸的制备及熔点测定

熔点测定是初步定性检测、鉴定有机化合物的基本方法, 我们采用Perkin反应制备肉桂酸, 经重 结晶提纯干燥后, 用显微熔点测定仪测定肉桂酸的熔点。同时, 提供一个未知样(事先不告诉学生是 什么样品), 让学生测定其熔点, 从而把熔点测定基本操作融入到实际产品的检验中, 改变以往只提 供一个已知样品的做法, 增强了学生的学习兴趣。

实例八: 1-溴丁烷的制备

本实验步骤较多, 操作过程较长, 要让学生领会实验的操作要点, 整个实验就会比较顺利完 成 ${ }^{[15]}$ 。

(1) 硫酸的浓度: 制备1-溴丁烷通常采用 $\mathrm{NaBr}-\mathrm{H}_{2} \mathrm{SO}_{4}$ 法。本实验中, $\mathrm{H}_{2} \mathrm{SO}_{4}$ 浓度是一个非常重要 的因素。提高 $\mathrm{H}_{2} \mathrm{SO}_{4}$ 浓度, 加热回流时有大量的 $\mathrm{HBr}$ 气体从冷凝管顶端逸出生成酸雾, 对 $\mathrm{HBr}$ 的氧化 性也增强。降低硫酸的浓度, 可使逸出的 $\mathrm{HBr}$ 气体大大减少, 但硫酸浓度如果太低, 不利于醇的质子 化, 影响亲核取代反应的进行。研究发现, 本实验采用 $63.5 \%$ 的 $\mathrm{H}_{2} \mathrm{SO}_{4}$ (10 mL浓硫酸 $+10 \mathrm{~mL}$ 水), 加 热回流时, 基本上没有 $\mathrm{HBr}$ 气体从冷凝管顶端逸出。硫酸配制以后, 一定要充分冷却, 否则加入到反 应瓶后, 颜色即会加深。

(2) 加热回流: 本实验学生产品收率不高, 一般在 $30 \%-40 \%$, 因此, 要规范操作, 尽可能减少损 失。对反应温度来说, 开始反应时不要太高, 否则反应生成的 $\mathrm{HBr}$ 来不及反应就会逸出, 可以明显地 看到用于气体吸收的烧杯中的水有气流波动, 检测水的酸性很强, 另外, 反应混合物的颜色也会很 快变深, 操作情况良好时, 反应瓶中油层呈浅黄色, 如果油层为棕色或棕黑色, 说明副反应较为严 重。过高的反应温度也有利于1-丁醇分子脱水生成1-丁烯、正丁醚。

在加热回流过程中逐渐分成油层、水层两相。如果回流温度过高, 因发生氧化还原反应生成 $\mathrm{Br}_{2}$, 油层的颜色由淡黄色(黄色)逐渐变成棕色(深棕色)。随着反应的进行, 颜色又会变浅一些, 变成黄色 (淡黄色), 其原因除了部分 $\mathrm{Br}_{2}$ 挥发逸出外, 还有可能是 $\mathrm{Br}_{2}$ 与副产物 1 -丁烯发生了加成反应, 使颜色 裉去。

(3) 粗蒸馏: 馏出液分两层, 通常下层为粗1-澳丁烷, 上层为水相, 若未反应的正丁醇较多及生 成较多的副产物丁醚, 或因蒸馏过久蒸出一些溴化氢恒沸物, 则液层的相对密度发生变化, 油层可 能悬浮或变为上层, 遇此现象, 可加清水稀释使油层下沉。

\section{3 结语}

教学改革, 教学相长, 取得了应有的成效。得益于有机化学实验教学内容的改革, 学生有了基 
本的研究创新思维和较强的动手能力, 有利于实验教学向第二课堂延伸, 将新开发的综合性实验、 研究探索性实验等应用到大学生课外科技活动, 让学生带着问题进入实验室, 第二课堂与第一课堂 齐头并进, 促进学生多样化成才。我校学生在全国高校化学学科面向本科生的各类学科竞赛中均取 得了优良的成绩, 也为学生继续深造和工作打下了坚实的基础。同时, 实验教学改革极大地提高了 教师的教学和教研水平, 已获得了多项省部级教学成果奖, 编辑出版的《新编基础化学实验II-有机 化学实验》教材先后被评为浙江省高校 “十二五” 优秀教材、“十三五” 新形态教材, 被多所省内外 高校采用作为教学用书, 并有力地支撑了基础化学实验国家精品、国家级精品资源共享课程、有机 化学实验省级一流课程以及化学化工国家级实验教学示范中心的建设。当然, 套用一句老话, 改革 永远在路上, 实验教学改革是永无止境的, 包括实验教学的内容、教学方法、教学手段等, 随着科 学技术的日新月异, 都应当与时俱进, 以不断地适应乃至超前于人才培养的需要。

\section{参 考 文 献}

[1] 夏建国, 赵军. 高等工程教育研究, 2017, No. 3, 15 .

[2] 陈世清. 武汉工程大学学报, 2010, 32 (10), 100.

[3] 强根荣, 曾秀琼, 杨振平, 刘秋平, 艾宁. 实验室研究与探索, 2018, 37 (9), 236.

[4] 罗志勇, 李泽全, 张胜涛, 周晓梅, 邓银. 实验室研究与探索, 2009, 28 (4), 122.

[5] 强根荣, 王海滨, 王红, 杨振平, 施仁信. 实验室研究与探索, 2020, 39 (9), 162.

[6] 孙浩, 李辉. 中国大学教学, 2015, No. 4, 78 .

[7] 强根荣, 王红, 杨振平. 实验室研究与探索, 2020, 39 (4), 182.

[8] 石磊, 杨红兵. 化学教育, 2016, 37 (8), 29.

[9] 周金梅, 林敏, 杨俐锋. 厦门大学学报(自然科学版), 2003, 42 (5), 679.

[10] 丁成, 倪金平, 唐荣, 马狄, 强根荣. 浙江工业大学学报, 2009, 37 (5), 542.

[11] 强根荣, 金红卫, 盛卫坚. 新编基础化学实验II-有机化学实验. 第3版. 北京: 化学工业出版社, 2020: 9 .

[12] 杨振平, 王海滨, 孙莉, 王红, 强根荣. 实验技术与管理, 2011, 28 (12), 141.

[13] 李厚金, 赖瑢, 朱可佳, 张利, 陈六平. 大学化学, 2017, 32 (4), 21.

[14] 王红, 刘秋平, 王海滨, 杨振平, 强根荣. 实验室研究与探索, 2019, 38 (7), 151.

[15] 强根荣, 孙莉, 王海滨, 裴文, 杨振平. 实验室研究与探索, 2013, 32 (11), 180. 